\title{
AN EXACT EQUATION FOR THE FREE SURFACE OF A FLUID IN A POROUS MEDIUM*
}

\author{
WILLIAM ARTILES ${ }^{\dagger}$ AND ROBERTO A. KRAENKEL ${ }^{\dagger}$
}

\begin{abstract}
We study the problem of the evolution of the free surface of a fluid in a saturated porous medium, bounded from below by a flat impermeable bottom, and described by the Laplace equation with moving-boundary conditions. By making use of a convenient conformal transformation, we show that the solution to this problem is equivalent to the solution of the Laplace equation on a fixed domain, with new variable coefficients, the boundary conditions. We use a kernel of the Laplace equation which allows us to write the Dirichlet-to-Neumann operator, and in this way we are able to find an exact differential-integral equation for the evolution of the free surface in one space dimension. Although not amenable to direct analytical solutions, this equation turns out to allow an easy numerical implementation. We give an explicit illustrative case at the end of the article.
\end{abstract}

Key words. free surface evolution, flow in porous media, mathematical modeling, conformal transformation, Dirichlet-to-Neumann, groundwater flow

AMS subject classifications. 35S30, 35Q35, 76S05

DOI. $10.1137 / 050644835$

1. Introduction. In this work we shall address a conceptually simple, yet until now not fully solved, question: given a fluid totally contained in a homogeneous, saturated, porous medium, bounded from below by a flat impermeable bottom and with a free deformable surface above, write down the evolution equation for the free surface in the case where the fluid can be considered two-dimensional and unbounded in the horizontal direction.

This is a classical problem. It is mathematically expressed by the Laplace equation in two dimensions, with boundary conditions on an unknown, time-dependent, boundary. As will be clear from the equations in the next section, its solution corresponds to the determination of a Dirichlet-to-Neumann operator. The usual way to tackle it is by a perturbative approach. Small parameters are introduced, measuring the relative amplitude of the motion and the longness of the perturbation:

$$
\alpha=\frac{a}{h_{0}}, \quad \beta=\left(\frac{h_{0}}{\lambda}\right)^{2},
$$

where $a$ is the amplitude of the surface displacement, $h_{0}$ is the unperturbed depth, and $\lambda$ is the typical wavelength of the perturbation. When $\beta \ll 1$ we have the Dupuit approximation, corresponding to the physical assumption of hydrostatic motion. Its use, together with the Darcy law, leads through an asymptotic expansion to the Boussinesq equation for the total thickness of the fluid [1], which in convenient nondimensional variables (see below) reads simply

$$
h_{t}=\left(h_{x} h\right)_{x}
$$

\footnotetext{
${ }^{*}$ Received by the editors November 10, 2005; accepted for publication (in revised form) November 28, 2006; published electronically February 23, 2007.

http://www.siam.org/journals/siap/67-3/64483.html

†Instituto de Física Teórica, Universidade Estadual Paulista - UNESP, R. Pamplona 145, 01405900 São Paulo, Brazil (william@ift.unesp.br, kraenkel@ift.unesp.br). The research of the first author was supported by FAPESP, São Paulo. The research of the second author was partially supported by CNPq/Brazil.
} 
Equation (1.2) has been widely studied, not only in the context of porous media dynamics, but in such areas as high temperature gas dynamics [2] or convective instabilities [3]. Although not an integrable equation, its long-time behavior is known, being dominated by the self-similar solution, for localized initial data [4]. These selfsimilar solutions exhibit shocks, that is, propagating regions where the first derivative is singular. One speaks of diffusive waves to characterize these solutions.

Extensions of the Boussinesq equation have been proposed, in the same perturbation theoretic spirit, by several authors, encompassing higher order expansions in the longness parameter $[5,6,7]$ together with a small $\alpha[8]$. Still other works introduce a new perturbative parameter, the steepness $\alpha \sqrt{\beta}$ [9]. For localized initial conditions, the above-mentioned shocks become smoothed out, and we have propagating fronts.

A great analogy exists between the present problem and the determination of the evolution of a free surface of an inviscid fluid, the water-wave problem with the same two-dimensional geometry. Again, one studies the two-dimensional Laplace equation with a free boundary, but with different boundary conditions, and the determination of the evolution of the free surface is again equivalent to the determination of a Dirichlet-to-Neumann operator. Perturbative expansions have been widely used, dating back to the nineteenth century [10]. The same parameters $\alpha$ and $\beta$ as above come into play. Assuming $\alpha \ll 1$ and $\beta \ll 1$ with $\mathcal{O}(\alpha) \approx \mathcal{O}(\beta)$ results in the asymptotic theory named long waves in shallow water and described by the Boussinesq system of equations, or the Benney-Luke equations [11], or the Korteweg-de Vries equation (for waves in a given direction) and its asymptotic equivalents, like the Kaup-Boussinesq [12] or the Benjamin-Bona-Mahoney-Peregrine equations [13, 14]. Alternatively, one can also directly expand the Dirichlet-to-Neumann operator in a Fourier series (due to a result on the analyticity of the Dirichlet-to-Neumann operator given in [15]), leading to numerically efficient integration schemes [16, 17]. So-called fully dispersive waves, where no assumption on $\beta$ is made, have been obtained by making use of properties of harmonic functions, which is natural in the context of the two-dimensional Laplace equation, in [18]. Extensions to waves over variable topography have been obtained in [19] by using results on an analytical representation of the Dirichlet-to-Neumann operator given in [20]. All these results explore the smallness of one or two parameters in order to obtain an approximate expression for evolution of the free surface. On a different path, an important nonperturbative result was obtained in [21], where an exact integral-differential equation for the evolution of the free surface was obtained after the introduction of convenient conformal mappings. This equation was numerically studied by an FFT pseudospectral method in [22]

In the present work we will present an exact integral-differential equation for the evolution of a free surface in a porous medium which is analogous to the results obtained in $[21,22]$ for the water-wave problem, although we will follow some different steps from these papers. We will take advantage of a conformal map, mapping the region filled with fluid to a straight strip, thus transforming the free surface problem to a fixed domain problem for the Laplace equation, but with transformed boundary conditions, which, however, will be explicitly solvable. Although not promptly amenable to analytical calculations, the equation will lend itself to the implementation of an efficient numerical method.

The study of free surface dynamics in a porous medium finds its main applications in the investigations of groundwater oscillations in unconfined aquifers in coastal regions. In such regions, the fluctuations of the sea surface, in the form of either surface waves or tidal oscillations, induce watertable oscillations. These oscillations, in turn, affect the environmental dynamics in the region. Many works have addressed this 
question $[5,6,7,8,9,23]$ on the theoretical side, providing equations to be used in larger integrated models for coastal environments. In particular, we should mention the effect caused by the periodic, tidal-induced, variation of the sea level, which is to induce a watertable over height with respect to the mean sea level. Our numerical calculations at the end of this article will illustrate this point.

2. Governing equations. The formulation of the problem is standard and can be found in textbooks $[1,24]$. We place ourselves in a two-dimensional plane geometry. Let us call $y$ the vertical axis, defined by gravity's direction, and $x$ the perpendicular, horizontal, direction. Consider a fluid filling a porous medium, lying over a flat impermeable bottom, up to a total height limited by a free surface given by a curve described by $y=h(x)$. The relevant dynamical variable is the piezometric head $\Phi(x, y, t)$, defined as

$$
\Phi=\frac{P}{\gamma}+y,
$$

where $P$ is the pressure and $\gamma=\rho g$ the specific weight. We assume Darcy's law; that is, we suppose that the seepage velocity is proportional to the gradient of the piezometric head, i.e,

$$
\mathbf{u}=-K \nabla \phi
$$

where $K$ is the permeability of the medium. Darcy's law is valid for the situation we have in mind, which is the flow of water percolating in rocks and soils. Theoretically it can be obtained from Stokes flow together with asymptotic expansions in a parameter measuring the ratio of microscopic (pores) length scales to macroscopic ones. NonDarcian effects would typically arise if the flow in pores became turbulent (e.g., in high-rate gas wells).

Supposing the validity of Darcy's law and taking the flow as incompressible, we come to our dynamical equation

$$
\Phi_{x x}+\Phi_{y y}=0, \quad 0<y<h(x, t),
$$

with the boundary conditions at the free surface given by

$$
\begin{gathered}
\Phi=h-h_{0} \quad \text { at } \quad y=h(x, t), \\
h_{t}-\frac{K}{n_{e}} \Phi_{x} h_{x}+\frac{K}{n_{e}} \Phi_{y}=0 \quad \text { at } \quad y=h(x, t),
\end{gathered}
$$

where $n_{e}$ is the effective porosity and the displacement surface $h-h_{0}$ is an integrable function. At the bottom, we have a Neumann condition:

$$
\Phi_{y}=0, \quad y=0 .
$$

The problem is posed with an initial condition for the free surface, $h(x, 0)=\varphi(x)$. The reader will appreciate here that the above equations are directly connected to the Dirichlet-to-Neumann operator. In rescaled variables, (2.3) says that the timederivative of $h(x, t)$ is proportional to the normal derivative of $\Phi(x, y, t)$ at the surface $y=h(x, t)$. We have thus the Laplace equation with a Dirichlet condition at the free boundary, (2.2) (in terms of the unknown function $h(x, t)$ ), and we have to find the normal derivative of the solution at this boundary (again in terms of $h(x, t)$ ) to insert 
it into (2.4), implying an evolution equation for the free surface, $h(x, t)$. Therefore, the solution to our problem goes through a Dirichlet-to-Neumann operator. We will, however, avoid explicitly introducing it here, for the sake of simplicity and as we would not really gain much in doing so. Let us also point out here that the main difference between the equations governing the classical water-wave problem and those that govern the porous medium problem under consideration can be seen in (2.2), which in the last case is much simpler than in the former case, where it involves timederivatives and nonlinear terms.

Our strategy to broach the problem will be the following: (i) first introduce nondimensional variables; (ii) next, define a conformal transformation from the strip $\Re \times(0, h(x))$ to $\Re \times(0, \mu)$, where $\mu$ is a constant to be defined below; (iii) this transformation eliminates the free-boundary problem, replacing it by a Laplace equation with new boundary conditions, involving the Jacobian of the transformation; (iv) we then solve the Laplace equation, with mixed Neumann-Dirichlet conditions, in terms of the unknown function describing the free surface, resulting in an equation for this surface, in conformal coordinates; (v) once this equation is obtained, we will develop an asymptotic analysis in a parameter measuring the longness of the wave with respect to the depth and obtain classical results on the problem; (iv) we close the paper with some numerical results on the full equations for the free surface.

3. Nondimensional equations and conformal transformation. We first write (2.1)-(2.4) in a nondimensional form. To do so, we introduce the following nondimensional variables:

$$
\begin{aligned}
& x=\lambda x^{\prime}, \quad y=\lambda y^{\prime}, \quad h=h_{0} h^{\prime}\left(x^{\prime}, t^{\prime}\right), \\
& \Phi=h_{0} \Phi^{\prime}, \quad t=\frac{n_{e}}{K} \frac{\lambda^{2}}{h_{0}} t^{\prime},
\end{aligned}
$$

where $\lambda$ is the typical wavelength of the free-surface perturbation and $h_{0}$ is the undisturbed depth of the fluid. In these new variables, we come to the following system of equations:

$$
\begin{aligned}
\Delta \Phi & =0, & 0<y & <\mu h(x, t), \\
\Phi & =h-1, & y & =\mu h(x, t), \\
0 & =h_{t}-\Phi_{x} h_{x}+\frac{1}{\mu} \Phi_{y}, & y & =\mu h(x, t), \\
\Phi_{y} & =0, & y & =0,
\end{aligned}
$$

where all primes have been omitted for notational convenience. A dimensionless parameter appears in these equations, $\mu=h_{0} / \lambda$. This would be the usual perturbative parameter for long-wave asymptotics (Dupuit approximation), where $\mu \ll 1$, meaning that the wavelength is much larger than the depth. We will not make this approximation from the beginning. Instead, we will obtain an exact equation for the free surface and only then take $\mu \ll 1$ in order to rederive previously known equations.

Further, we should note that we used a nondimensional variable so as to preserve the Laplacian, a fact of which we will make good use in what follows.

The crucial step in our procedure is the introduction of a conformal mapping. Consider a strip in the $w$-plane, $w=\xi+i \zeta$, given by $\Re \times[0, \mu]$. A mapping of this strip to the undulated strip in the $z$-plane, $z=x+i y$, given by $\Re \times[0, \mu h(x(\xi, \mu), t)]$, 
is defined as a harmonic function, given as the solution of the Dirichlet problem

$$
\begin{aligned}
& y_{\xi \xi}+y_{\zeta \zeta}=0, \\
& y(\xi, \mu)=\mu h(x(\xi, \mu), t), \\
& y(\xi, 0)=0
\end{aligned}
$$

if we suppose that we know the function $x(\xi, \mu)$ in the time $t$.

Time $t$ plays the role of a parameter in these equations: for each $t$ we have different functions $x(\xi, \zeta)$ and $y(\xi, \zeta)$. Equations (3.5)-(3.7) can be solved explicitly. Indeed, the solution is given by the imaginary part of

$$
z(w)=\frac{1}{2} \int_{-\infty}^{\infty} \tanh \left[\frac{\pi}{2 \mu}\left(w-\xi^{\prime}\right)\right] h\left(x\left(\xi^{\prime}, \mu\right), t\right) d \xi^{\prime} .
$$

Equation (3.5) is verified trivially, as well as (3.7). To show that (3.6) is also satisfied, we first obtain explicitly the imaginary part of (3.8). This is easily done with the help of the trigonometric identity

$$
\tanh \left[\frac{\pi}{2 \mu}\left(w-\xi^{\prime}\right)\right] \frac{\sinh \left[\frac{\pi}{\mu}\left(\xi-\xi^{\prime}\right)\right]+i \sin \left[\frac{\pi}{\mu} \zeta\right]}{\cosh \left[\frac{\pi}{\mu}\left(\xi-\xi^{\prime}\right)\right]+\cos \left[\frac{\pi}{\mu} \zeta\right]},
$$

which implies that

$$
y(w)=\frac{1}{2} \int_{\Re} \frac{\sin \left[\frac{\pi}{\mu} \zeta\right] h\left(\xi^{\prime}, t\right)}{\cosh \left[\frac{\pi}{\mu}\left(\xi-\xi^{\prime}\right)\right]+\cos \left[\frac{\pi}{\mu} \zeta\right]} d \xi^{\prime} .
$$

We now use the fact that the convolution between two functions is equal to the inverse Fourier transform of the product of their Fourier transform, $\mathcal{F}^{-1}[\mathcal{F}(f) \star \mathcal{F}(g)]=f \star g$, and obtain that, after some algebra,

$$
\begin{aligned}
y(w) & =\mu \int_{\Re} \frac{\sinh [2 \pi k \zeta]}{\sinh [2 \pi k \mu]} \mathcal{F}[h] e^{2 \pi i \kappa \xi} d \kappa \\
& =\mu \int_{\Re} \frac{\sinh [2 \pi k \zeta]}{\sinh [2 \pi k \mu]} \mathcal{F}[h-1] e^{2 \pi i \kappa \xi} d \kappa+\zeta .
\end{aligned}
$$

Evaluated at $\zeta=\mu$, (3.11) gives (3.6) immediately. Therefore, we now have transformed our physical space, moving-boundary, domain to a fixed one through a timedependent conformal mapping which is explicitly given by either (3.10) or (3.11).

4. Transformed equations and their solution: Free-surface evolution. Although the conformal transformation introduced in the last section leaves the Laplacian in (3.1) invariant, this is not so for the boundary conditions. In the new coordinates $(\xi, \zeta)$ the system given by (3.1)-(3.4) takes, nevertheless, a simple and convenient form. If we use that, at the upper surface, $y=\mu h(x(\xi, \mu), t)$, $\partial_{\zeta}=x_{\xi}\left(\partial_{y}-\mu h_{x} \partial_{x}\right)$, which follows from the Cauchy-Riemann conditions, $x_{\xi}=y_{\zeta}$ and $x_{\zeta}=-y_{\xi}$ and (3.6), we come to the transformed equations

$$
\begin{aligned}
\Delta \Phi & =0, & 0<\zeta & <\mu, \\
\Phi & =h-1, & \zeta & =\mu, \\
0 & =h_{t}+\frac{\Phi_{\zeta}}{\mu x_{\xi}}, & \zeta & =\mu, \\
\Phi_{\zeta} & =0, & \zeta & =0 .
\end{aligned}
$$


This is now a system of equations defined on a fixed domain, with a coordinatedependent coefficient in (4.3). The system of equations formed by (4.1), (4.2), (4.4) may now be seen as a Laplace equation to be solved with a Neumann condition at $\zeta=0$ and a Dirichlet condition at $\zeta=\mu$, where $h-1$ is the prescribed boundary value of $\Phi(\xi, \zeta)$. A solution to this problem reads

$$
\Phi(\xi, \zeta, t)=\int_{-\infty}^{\infty} \mathcal{F}[\Phi(\xi, \mu, t)] \frac{\cosh [2 \pi \kappa \zeta]}{\cosh [2 \pi \kappa \mu]} e^{2 \pi i \kappa \xi} d \kappa,
$$

where $\mathcal{F}[\Phi](\kappa, \mu, t)$ is the Fourier transform of the piezometric head $\Phi$ at the surface $\zeta=\mu$, given in terms of $h-1$.

Equation (4.5) solves (4.1), (4.2), (4.4). We may use it to obtain a relation between $\Phi_{\zeta}$ and $\Phi$ at the surface (having thus implicitly constructed a Dirichlet-to-Neumann operator):

$$
\begin{aligned}
\Phi_{\zeta}(\xi, \mu, t) & =\int_{-\infty}^{\infty} 2 \pi \kappa \tanh [2 \pi \kappa \mu] \mathcal{F}[\Phi] e^{2 \pi i \kappa \xi} d \kappa \\
& =\int_{-\infty}^{\infty}-i \tanh [2 \pi \kappa \mu] \mathcal{F}\left[\Phi_{\xi}\right] e^{2 \pi i \kappa \xi} d \kappa \equiv \mathbf{T} \partial_{\xi}[\Phi],
\end{aligned}
$$

where $\mathbf{T}[-]$ is an integral operator defined by the above equation. It will be quite useful in the numerical calculations. For the moment, it is introduced for notational convenience. Inserting the above equation into (4.3) gives

$$
h_{t}+\frac{1}{\mu x_{\xi}} \mathbf{T} \partial_{x}[h]=0,
$$

where use was made of the fact that $h_{\xi}=\Phi_{\xi}$ at $\zeta=\mu$. Equation (4.8) gives the time evolution of the free surface in the conformal coordinates $(\xi, \zeta)$. We should, however, note that $x_{\xi}$, which can be derived from (3.8), depends also on $h(x, t)$, making (4.8) nonlinear.

We can see the system formed by (3.8) and (4.8) as determining the time evolution of the free surface exactly. Let us, however, write it in a more compact form. If we note that the real part of (3.8) may be expressed as

$$
\begin{aligned}
x(\xi, \zeta) & =-i \mu \int_{-\infty}^{\infty} \frac{\cosh [2 \pi \kappa \zeta]}{\sinh [2 \pi \kappa \mu]} \mathcal{F}[h] e^{2 \pi i \kappa \xi} d \kappa \\
& =-i \mu \int_{-\infty}^{\infty} \frac{\cosh [2 \pi \kappa \zeta]}{\sinh [2 \pi \kappa \mu]} \mathcal{F}[h-1] e^{2 \pi i \kappa \xi} d \kappa+\xi,
\end{aligned}
$$

where we again used (3.9) and the properties of the Fourier transform of the convolution of two functions, we can obtain $x_{\xi}$ in the limit $\zeta \rightarrow \mu$ :

$$
x_{\xi}=\mu \int_{-\infty}^{\infty} 2 \pi \kappa \operatorname{coth}[2 \pi \kappa \mu] \mathcal{F}[h] e^{2 \pi i \kappa \xi} d \kappa
$$

or

$$
x_{\xi}=-\mu \mathbf{T}^{-1} \partial_{\xi}[h]
$$

The evolution equation for the free surface is thus given in the conformal coordinates by

$$
h_{t}=\frac{1}{\mu^{2}} \frac{\mathbf{T} \partial_{\xi}[h]}{\mathbf{T}^{-1} \partial_{\xi}[h]}=0
$$


The above equation displays the time evolution of the free surface elegantly, although one could object that it could be difficult to use it in actual analytical calculations. We will, therefore, go further and show two distinct developments originating from (4.12): asymptotics and numerics.

5. Long-wave asymptotics. The theory of long-wave perturbation for a fluid in a porous medium is a classical subject, which has been extensively studied in many different aspects. Here we will systematically rederive this approximation from the exact equation (4.12), or equivalently, from (4.8), (4.11). It corresponds to the limit $\mu \ll 1$.

To proceed, we note first the identities

$$
\begin{aligned}
\mathbf{T} \partial_{\xi}[h] & =\int_{-\infty}^{\infty} 2 \pi \kappa \tanh [2 \pi \kappa \mu] \mathcal{F}[h] e^{2 \pi i \kappa \xi} d \kappa=-\tan \left(\mu \partial_{\xi}\right) \partial_{\xi}[h], \\
\mathbf{T}^{-1} \partial_{\xi}[h] & =-\int_{-\infty}^{\infty} 2 \pi \kappa \operatorname{coth}[2 \pi \kappa \mu] \mathcal{F}[h] e^{2 \pi i \kappa \xi} d \kappa=-\cot \left(\mu \partial_{\xi}\right) \partial_{\xi}[h],
\end{aligned}
$$

where the tan and cot are defined by their series. Equations (4.8), (4.11) become respectively

$$
\begin{aligned}
& \mu h_{t} x_{\xi}-\tan \left(\mu \partial_{\xi}\right) \partial_{\xi}[h]=0, \\
& x_{\xi}=\mu \cot \left(\mu \partial_{\xi}\right) \partial_{\xi}[h] .
\end{aligned}
$$

Introduce now expansions up to order $\mu^{2}$ of both equations. This implies

$$
\begin{gathered}
0=h_{t} x_{\xi}-h_{\xi \xi}-\frac{\mu^{2}}{3} h_{\xi \xi \xi \xi}+\cdots, \\
x_{\xi}=h-\frac{\mu^{2}}{3} h_{\xi \xi}+\cdots
\end{gathered}
$$

The derivatives $h_{\xi}$ may be rewritten iteratively as terms of $h_{x}$ in an asymptotic sense:

$$
h_{\xi}=h_{x} x_{\xi}=h_{x} h-\frac{\mu^{2}}{3} h_{x} h_{\xi \xi}+O\left(\mu^{4}\right),
$$

and then

$$
\begin{aligned}
h_{\xi \xi} & =\left(h_{x} h\right)_{\xi}-\frac{\mu^{2}}{3}\left(h_{x} h_{\xi \xi}\right)_{\xi}+O\left(\mu^{4}\right) \\
& =\left(h_{x} h\right)_{x} x_{\xi}-\frac{\mu^{2}}{3}\left(h_{x} h_{\xi \xi}\right)_{x} x_{\xi}+O\left(\mu^{4}\right), \\
h_{\xi \xi \xi \xi} & =\left(h_{\xi \xi \xi}\right)_{x} x_{\xi}+O\left(\mu^{2}\right),
\end{aligned}
$$

from which we obtain, by substituting into (5.5), the following:

$$
\begin{aligned}
h_{t} & =\left(h_{x} h\right)_{x}-\frac{\mu^{2}}{3}\left(h_{x} h_{\xi \xi}\right)_{x}+\frac{\mu^{2}}{3}\left(h_{\xi \xi \xi}\right)_{x}+\cdots \\
& =\left(h_{x} h\right)_{x}+\frac{\mu^{2}}{3}\left[-h_{x} h_{\xi \xi}+h_{\xi \xi x} x_{\xi}\right]_{x}+\cdots \\
& =\left(h_{x} h\right)_{x}+\frac{\mu^{2}}{3}\left[-2 h_{x} h_{\xi \xi}+h_{x} h_{\xi \xi}+h_{\xi \xi x} h\right]_{x}+\cdots
\end{aligned}
$$




$$
\begin{aligned}
& =\left(h_{x} h\right)_{x}+\frac{\mu^{2}}{3}\left[-2 h_{x} h_{\xi \xi}+\left(h_{\xi \xi} h\right)_{x}\right]_{x}+\cdots \\
& =\left(h_{x} h\right)_{x}+\frac{\mu^{2}}{3}\left[-2 h_{x}\left(h_{x} h\right)_{x} h+\left(\left(h_{x} h\right)_{x} h^{2}\right)_{x}\right]_{x}+\cdots \\
& =\left(h_{x} h\right)_{x}+\frac{\mu^{2}}{3}\left[-2 h_{x} h\left(h_{x} h\right)_{x}+\left(h_{x x} h^{3}\right)_{x}+\left(h_{x}^{2} h^{2}\right)_{x}\right]_{x}+\cdots .
\end{aligned}
$$

Thus, to order $\mu^{2}$, we have the equation

$$
h_{t}=\left(h_{x} h\right)_{x}+\frac{\mu^{2}}{3}\left[h_{x x} h^{3}\right]_{x x}+\cdots,
$$

which had been derived in [7]. It is quite evident that we could consistently continue the expansion to any desired order. Also, one notes that the approximation of small amplitude fluctuations was not made, but could consistently be introduced, as long as we previously state the order relations between $\mu^{2}$ and the order of magnitude of the amplitude fluctuations. A further point here is to again mention the analogous problem for water waves. Equation (5.11) shows us that the problem at hand is, phenomenologically speaking, intrinsically diffusive. The first term in (5.11) represents a nonlinear diffusion, as if the diffusion coefficient were proportional to $h$, and the next terms are higher order and nonlinear diffusion ones. Water waves offer a comparison if one exchanges diffusion for dispersion.

6. The linear problem. In the last section we saw that it is possible to obtain a perturbative expansion in the wavelength parameter $\mu$, giving rise to a nonlinear partial differential equation, even in the lowest order. In this section we will explore another possibility, which is to leave $\mu$ free, and obtain a new expansion based on the smallness of the amplitude of the surface elevation. The corresponding lowest order equation is a linear partial integro-differential equation, whose solution we will also present.

Let us go back to (4.12), and let us write $h=1+\eta$, where $\eta$ is the displacement of the free surface from its undisturbed position. In the case where $\eta \ll 1$, as a first approximation we can obtain a differential equation for $\eta$ by noting that

$$
\begin{aligned}
\eta_{t} & =-\frac{1}{\mu} \frac{\mathbf{T}\left[\eta_{\xi}\right]}{1-\mu \mathbf{T}^{-1}\left[\eta_{\xi}\right]} \\
& =-\frac{1}{\mu} \mathbf{T}\left[\eta_{\xi}\right]-\mathbf{T}\left[\eta_{\xi}\right] \mathbf{T}^{-1}\left[\eta_{\xi}\right]+\cdots,
\end{aligned}
$$

and thus that the lowest order linear equation reads

$$
\eta_{t}=-\frac{1}{\mu} \mathbf{T}\left[\eta_{\xi}\right]
$$

In order to give the solution of (6.2) in a compact way, define the dispersion relation as

$$
w_{k}=\frac{2 \pi k}{\mu} \tanh [2 \pi k \mu],
$$

and the function $G(\xi, t)$ as the inverse Fourier transform of $e^{-w_{k} t}$,

$$
G(\xi, t)=\int_{\Re} e^{-w_{k} t} e^{2 \pi i k \xi} d k,
$$

where $G$ satisfies $\lim _{t \rightarrow 0} G(\xi, t)=\delta(\xi)$. 
This allows us to write the solution of (6.2), for $\xi \in \Re$, as

$$
\begin{aligned}
\eta(\xi, t) & =\int_{\Re} e^{-w_{k} t} \mathcal{F}[\varphi] e^{2 \pi i k \xi} d k \\
& =\int_{\Re} G_{t}\left(\xi-\xi^{\prime}\right) \varphi\left(\xi^{\prime}\right) d \xi^{\prime},
\end{aligned}
$$

where $\mathcal{F}[\varphi]$ is the Fourier transform of the initial free surface position $\eta(\xi, 0)=\varphi(\xi)$. In the case $\mu \rightarrow 0$ then $w_{k}=(2 \pi k)^{2}, G_{t}(\xi)$ is the Gaussian exponential, and the solution for $\eta$ is

$$
\eta(\xi, t)=\frac{1}{2 \sqrt{\pi t}} \int_{\Re} e^{-\frac{\left(\xi-\xi^{\prime}\right)^{2}}{4 t}} \varphi\left(\xi^{\prime}\right) d \xi^{\prime},
$$

a well-known formula for the small amplitude long-wave approximation. From the last two sections, it is clear that (4.8) may be used as starting point for other perturbative expansions involving relations between two perturbative parameters. As an example, one can substitute $h=1+\eta$ into (5.11), write $\eta=\epsilon \bar{\eta}$ with $\epsilon \ll 1$, and have a two-parameter asymptotic expansion.

7. Numerics. In this section we will briefly describe a pseudospectral numerical method used to integrate (4.12) and display an example calculation for the classical problem of the tide-induced over-height in unconfined aquifers.

A very important point is that we were able to reduce the dynamics of a bidimensional boundary problem with a free surface to a one-dimensional problem given by a differential-integral equation in an exact way. Although (4.12) is somewhat odd for analytical calculations, it is quite convenient for numerical implementation. We do not need tools like, for instance, boundary integral methods involving singular operators. The Fourier-like transforms that appear in the integral operators are by no means a problem, as they can be easily managed by FFTs, resulting in a method with spectral accuracy. Let us now give a definite example of implementation of the method. We shall solve (4.12) with a periodic boundary condition $h(0, t)=1+$ $\alpha \sin (w t)$ at $x=0$. This simulates the effect of ocean tides in contact with groundwater in a coastal aquifer, through an idealized vertical beach. We take $h_{x}(L, t)=0$ when $L \gg h_{0}$. This last condition allows us to make a periodic extension to the interval $2 L$ by introducing an adjunct forced boundary condition as

$$
h(0, t)=1+\alpha \sin (w t) \text { and } h(2 L, t)=1+\alpha \sin (w t)
$$

and, consequently, allowing the use of Fourier transform methods. In this model the parameter $\alpha$ gives a measure of the nonlinearity of the problem. As initial condition we take $h(x, 0)=1$.

At each time step, the periodic functions $\left(x, x_{\xi}, h\right)$ are expanded as discrete Fourier series in $\xi$ using the FFT, and the T-transform is computed in Fourier space. For example, $\mathbf{T}\left[h_{\xi}\right]$ of a function can be found via FFT after multiplying the Fourier coefficients of $h$ by $2 \pi \kappa \tanh [2 \pi \kappa \mu]$, as it follows from (4.7). In a similar way we may compute $\mathbf{T}^{-1}\left[h_{\xi}\right]$. After evaluating nonlinear terms in physical space, we advance the solution of (4.12) in time with a 4th order Runge-Kutta method.

We have worked with 516, 512, or 1024 spatial points. The spatial step size is chosen in a range between 0.01 and 0.1 . Usually we work with the time step $\Delta t=0.01$. We do not need a high-pass filter. 

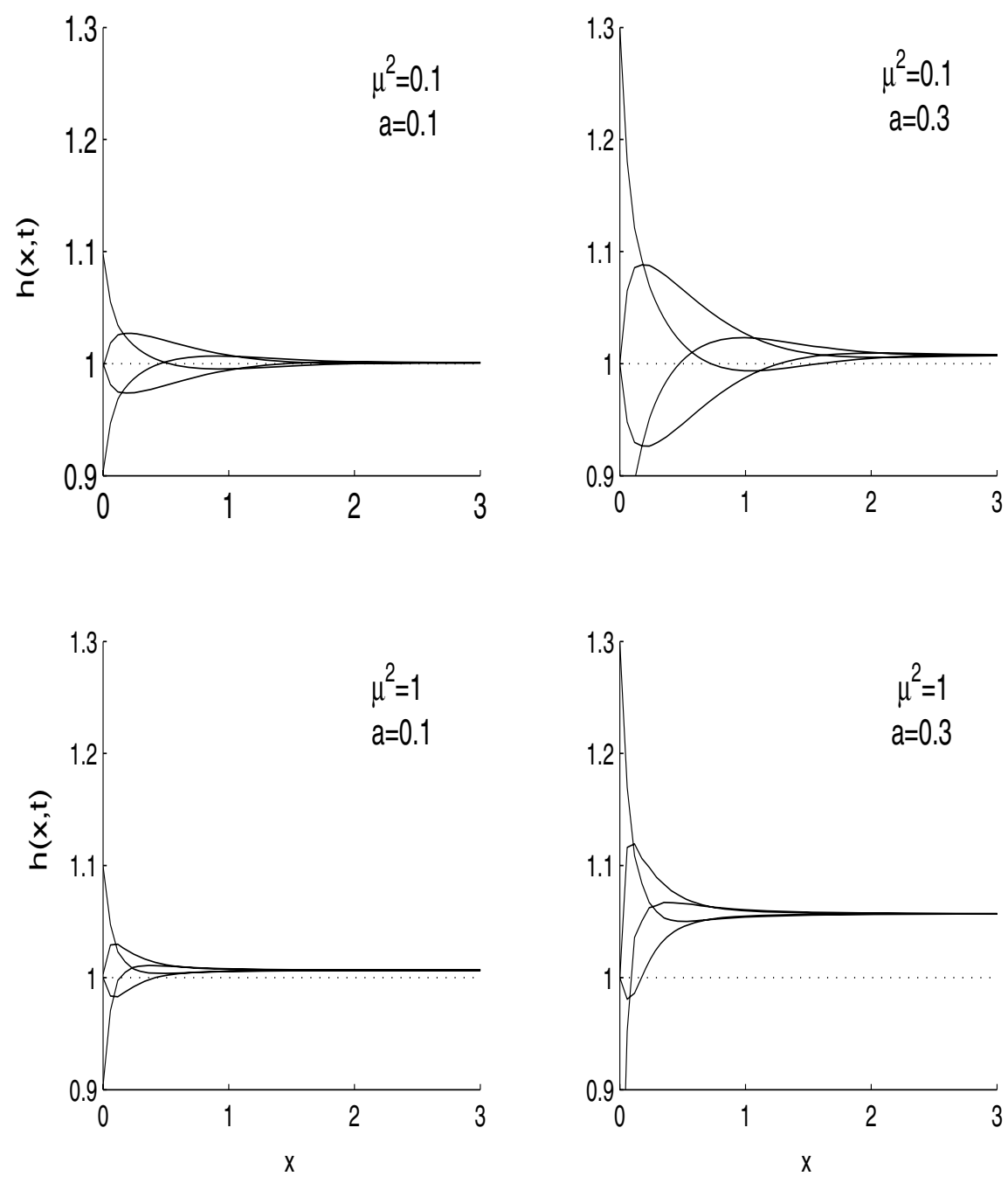

FIG. 7.1. Different profiles for the free surface when $t \rightarrow \infty$ and phase $w t=0, \frac{\pi}{2}, \pi, \frac{3 \pi}{2}$.

In Figure 7.1 we plot the surface profile for distinct values of $\alpha$ and $\mu$. The figure presents the numerical solution for a combination of two values of $\alpha$ and three values of $\mu$ when $t \rightarrow \infty$, and for four values the phase $w t=0, \frac{\pi}{2}, \pi, \frac{3 \pi}{2}$.

We see the free surface displacement decaying with the distance from the boundary $x=0$, while oscillating in both space and time. The decay rate is of the same order of magnitude of the parameter $\mu$, and the free surface elevation for large $x$ is of the order of $\alpha$.

8. Conclusions. We have introduced a new differential-integral equation exactly describing the evolution of a free surface of a fluid totally immersed in a saturated porous medium and bounded from below by an impermeable bottom. Our equation, (4.12), is a porous-media analogue of the exact equation found for water waves in [21] and numerically studied in [22]. We have also shown that the asymptotic long-wave 
expansion for this equations leads to known equations [7, 8]. The numerical implementation of (4.12) is easy, and we have provided a simple example.

The method that we have used is based on properties of harmonic functions and makes full use of a conformal transformation. Generalization to the case of an uneven bottom may follow quite easily and will be the object of future work. This should allow implementing calculations for more realistic cases of interaction of aquifers and tides through sloping beaches. A less evident generalization would be the extension of the results of this work for three-dimensional problems.

\section{REFERENCES}

[1] A. Fowler, Mathematical Methods in the Applied Sciences, Cambridge University Press, Cambridge, UK, 1997.

[2] Ya. B. Zeldovich e Yu. P., Physics of Shock Waves and High Temperature Hydrodynamic Phenomena, Vol. II, Academic Press, New York, 1967.

[3] R. A. Kraenkel, S. M. Kurcbart, J. G. Pereira, and M. A. Manna, Nonlinear diffusion process in a Bénard system at the critical point for the onset of convection, Phys. Rev. E, 47 (1993), pp. 3303-3306.

[4] E. S. SABInINA, Class of nonlinear degenerate parabolic equation, Doklady Acad. Nausk SSSR, 143 (1961), pp. 794-797.

[5] G. Dagan, Second order theory of shallow free surface flow in porous media, Quart. J. Mech. Appl. Math., 20 (1967), pp. 517-526.

[6] J.-Y. Parlange, F. Stagnitti, and J. L. Starr, Free surface flow in porous media and periodic solution of the shallow flow approximation, J. Hidrol., 70 (1984), pp. 251-263.

[7] P. Nielsen, R. Aseervatham, J. D. Fenton, and P. Perrochet, Groundwater waves in aquifers of intermediate depths, Adv. Water Resour., 20 (1996), pp. 37-43.

[8] P. LiU AND J. Wen, Nonlinear diffusive surface waves in porous media, J. Fluid Mech., 347 (1997), pp. 119-139.

[9] D.-S. Jenga, B. R. Seymour, D. A. Barry, J.-Y. Parlange, D. A. Lockingtone, and L. LI, Steepness expansion for free surface flows in coastal aquifers, J. Hydrology, 309 (2005), pp. $85-92$.

[10] G. B. Whitham, Linear and Nonlinear Waves, Wiley, New York, 1974.

[11] D. J. Benney and J. C. Luke, Interactions of permanent waves of finite amplitude, J. Math. Phys., 43 (1964), pp. 309-313.

[12] D. J. KAUP, Higher order water-wave equation and method for solving it, Prog. Theoret. Phys., 54 (1975), pp. 396-400.

[13] T. B. Benjamin, J. L. Bona, And J. J. Mahony, Model equations for long waves in nonlinear dispersive systems, Phil. Trans. Roy. Soc., A272 (1972), pp. 47-78.

[14] D. H. Peregrine, Long waves on a beach, J. Fluid Mech., 27 (1967), pp. 815-827.

[15] R. R. Coifman and Y. Meyer, Nonlinear harmonic analysis and analytic dependence, in Pseudodifferential Operators and Applications, Proceedings of Symposia in Pure Mathematics 43, F. Treves, ed., AMS, Providence, RI, 1985, pp. 71-79.

[16] W. Craig and C. Sulem, Numerical simulation of gravity waves, J. Comput. Phys., 108 (1993), pp. $73-83$.

[17] D. P. Nicholls and F. Reitich, Stability of high-order perturbative methods for the computation of Dirichlet-Neumann operators, J. Comput. Phys., 170 (2001), pp. 276-298.

[18] Y. Matsuno, Nonlinear evolutions of surface gravity-waves on a fluid of finite depth, Phys. Rev. Lett., 69 (1992), pp. 609-611.

[19] W. ARTiles AND A. NAChBin, Nonlinear evolution of surface gravity waves over highly variable depth, Phys. Rev. Lett., 93 (2004), paper 234501.

[20] P. Guidotti, A first-kind boundary integral formulation for the Laplace Dirichlet-to-Neumann map in 2D, J. Comput. Phys., 190 (2003), pp. 325-345.

[21] A. I. Dyachenko, V. E. Zakharov, and E. A. Kuznetsov, Nonlinear dynamics of the free surface of an ideal fluid, Plasma Phys. Rep., 22 (1996), pp. 829-840.

[22] Ya. A. Li, J. M. Hyman, AND W. Y. ChOI, A numerical study of the exact evolution equations for surface waves in water of finite depth, Stud. Appl. Math., 113 (2004), pp. 303-324.

[23] N. SU, F. LiU, AND V. ANH, Tides as phase-modulated waves inducing periodic groundwater flow in coastal aquifers overlaying a sloping impervious base, Environ. Model. Softw., 18 (2003), pp. 937-942.

[24] J. BeAr, Dynamics of Fluids in Porous Media, Dover, New York, 1988. 